\title{
The lateralized effects of concurrent cognitive and motor performance
}

\author{
K. McFARLAND and R. ASHTON \\ University of Queensland, St. Lucia, Queensland, Australia 4067
}

\begin{abstract}
Subjects were required to simultaneously tap two button switches, at two different distances apart, while at the same time performing a running memory span (RMS) task of either words or faces. Performance on the button-pressing task was assessed in terms of both speed and consistency. When the switches were close together, both of these measures showed leftand right-hand performance to be selectively disrupted by concurrent performance of faces and words memory tasks, respectively. With the switches further apart, selective disruption of the contralateral hand occurred only with the speed index. Using the consistency index, however, bilateral disruption was observed with both types of RMS tasks. Error and $d^{\prime}$ performance on the words and faces RMS tasks was only disrupted by contralateral manual activity. Some implications of these results for neuropsychological models of brain lateralization of function are discussed.
\end{abstract}

There is extensive evidence to indicate that the cerebral hemispheres are differentially involved in mediating certain human behaviors. The most wellknown observation is that the left hemisphere, of right-handed persons, usually mediates languagerelated cognitive activities, and that the right hemisphere usually mediates visuospatial, nonlanguage, cognitive activities (see Dimond \& Beaumont, 1974, for reviews).

There is also growing evidence that each cerebral hemisphere exerts bilateral control over different types of proximal motor activities. The findings of Boll (1974), Carmon (1970), Carmon and Benton (1969), and Fontenot and Benton (1971) suggest that the right hemisphere is specialized for tactileperceptual activity and for processing afferent kinesthetic sensation used to regulate movements in relation to space. Conversely, the findings of Kimura and Archibald (1974) and Wyke (1971) suggest that the left hemisphere is specialized, in some sense, for the bilateral control of repetitive and sequential movements of the hands and fingers.

A number of recent studies have been made of the relationship between cognitive and motor functional asymmetries in normal subjects by observing the effects that concurrent cognitive activity have upon left- and right-hand manual activity. In terms of the above evidence, it might be expected that a concurrent verbal task (for example) would produce a bilateral disruption of proximal motor activity. However, this is not found. Thus Lomas and Kimura

Reprint requests to $\mathbf{R}$. Ashton, Department of Psychology, University of Queensland, St. Lucia, Queensland, Australia 4067.
(1976) have demonstrated that a concurrent verbal task will disrupt right- but not left-hand performance on a motor task which requires rapid movement and repositioning of the hands or fingers (see also Hicks, 1975; Hicks, Provenzano, \& Rubenstein, 1975). To account for this finding, Lomas and Kimura (1976) suggest that bilateral disruption of the hands does not occur because those neural mechanisms of the left hemisphere which mediate the verbal task only overlap those which mediate right-hand repositioning and are functionally more distant from those that are used to mediate left-hand repositioning (see also Kimura, 1975).

McFarland and Ashton (Note 1) have also reported that a concurrent verbal task will disrupt rightbut not left-hand performance on a two-button tapping task. A further finding by these authors was that a concurrent visuospatial task will selectively disrupt left- but not right-hand performance on such a tapping task (see also McFarland \& Ashton, 1975). This finding may be interpreted by analogy to the Lomas and Kimura (1976) hypothesis mentioned above. Namely, the neural structures of the right hemisphere, which mediate the visuospatial cognitive task, may overlap only those right-hemisphere structures which mediate the spatial components of lefthand performance but not those structures which mediate the spatial components of right-hand performance.

McFarland and Ashton (Note 1) raised the possibility that the hypothesized differential overlap of the neural structures mediating the cognitive and motor tasks might be dependent upon a small load being imposed on these structures by the concurrent tasks. However, these authors examined this possibility 
only in the context of the load produced by the cognitive tasks. The effects of changing the load imposed by the manual task still needs to be examined. It is proposed to do this in the present study.

A two-button tapping task, similar to that used by McFarland and Ashton (Note 1) is used. However, it is modified such that the distance between the button switches may be either small or large. It was expected that selective disruption of the contralateral hand would be obtained with concurrent left- and right-hemisphere cognitive activity when the distance between the buttons was small. With the larger tapping distance, which increases the proximal activity (bilateral control) required to perform the task, it was expected that a bilateral disruption of the hands would be observed with the concurrent cognitive tasks.

\section{METHOD}

\section{Subjects}

Sixteen introductory psychology students served as subjects. All were right-handed as assessed by the Edinburgh Handedness Inventory (Oldfield, 1971) with laterality quotient scores above 65.

\section{Apparatus and Procedure}

The apparatus for the manual task consisted of two buttons mounted side by side on a box with the button surface slanted towards the subjects. The distance between the buttons could be varied from 6 to $12 \mathrm{~cm}$, which difference increased the Index of Difficulty (Fitts, 1954) by one bit.

The 16 subjects were randomly allocated to each of two groups, 8 in each group. One group was required to press the two button switches placed $6 \mathrm{~cm}$ apart, and the other group, the buttons placed $12 \mathrm{~cm}$ apart. The subjects were instructed to use either the left or right index finger (depending on the experimental condition) and to alternately press the two switches as fast and as consistently as possible at all times throughout the experiment, They received 7-8 min practice with each hand, and, apart from an initial familiarization period, they were not permitted to observe their manual performance. The buttons were connected to an Inter-data 5 minicomputer programmed to record the interresponse times (IRTs) of successive buttonpresses and to calculate the mean and the standard deviation of these IRTs for each experimental condition for each subject. The mean gives an index of speed performance, the standard deviation an index of consistency of performance. The cognitive tasks were running memory span tasks of 50 -item length (see Shepard \& Teghtsoonian, 1961; McFarland \& Ashton, Note 1). The first six items were always "new" items and the remaining were 22 new and 22 old items. The subject was required to respond old or new as each item was presented. Allocation of old and new items was random throughout the lists, with the restriction that an old item occur between 10 and 20 items (normally distributed with a mean of 15 ) after its first occurrence.

For the verbal (left-hemisphere) tasks, the items were selected randomly from the first 300 most frequently occurring four-letter words reported by Kučera and Francis (1967). The words were presented by means of an electronic alphanumeric light display unit, this unit being placed at eye level directly in front of the subject. In the visuospatial tasks, photographs of faces were used. Such stimuli are known to require predominantly right-hemisphere processing (Moscovitch, Sculion, \& Christie, 1976). All faces were randomly selected from a pool of 300 old and new pairs. The
300 faces were of students from a previous year, and all were unknown to the subjects of the experiment. The photographs were back-projected onto a screen $(8 \times 12 \mathrm{~cm})$, placed at eye level in front of the subject, by means of an automatic-change Carousel slide projector. When the light display unit presenting the words was not in use, it was removed from the line of sight of the subject so as not to obscure the screen on which the faces were presented. The presentation of the words and the slides was controlled by the minicomputer with the rate varying randomly between 1,500 and $3,000 \mathrm{msec}$ and a fixed exposure time of $800 \mathrm{msec}$. A fixed presentation rate was avoided, as this may have cued the subject on the number of buttonpresses to insert between successive items so as to maintain a consistent rate of tapping.

The subjects were given two practice runs with each of the words and faces memory tasks to ensure their familiarity with the stimulus and response requirements of the tasks. The two lists used for practice were selected randomly from each set of eight words and faces lists. Sixteen experimental conditions then followed. These consisted of the following eight conditions, each being completed twice: (1) words task alone; (2) faces task alone; (3) right hand alone; (4) left hand alone; (5) right hand with words task; (6) left hand with words task; (7) right hand with faces task; (8) left hand with faces task. The allocation of particular words and faces lists was random across conditions and subjects. The order of the 16 experimental conditions was randomized and semicounterbalanced within each group of eight subjects, with the restriction that no two conditions requiring the use of the same hand immediately follow each other. This was necessary in order to keep fatigue effects to a minimum. The subjects were also given 2-3 min rest between experimental conditions. The purpose of running each experimental condition twice was to ensure that a sufficient number of old and new trials were run to allow a signal detection theory (SDT) analysis of performance on the cognitive tasks to be carried out (Lockhart \& Murdock, 1970). The subjects were informed that the first six items were always new, and their responses to these were dropped from subsequent analysis. Thus, 44 old and 44 new trials were available for SDT analysis, which ensured a reasonable stability of the indices $d^{\prime}$ and $\beta$ (see McNicol, 1972).

For the concurrent task conditions, the subject was instructed to begin tapping the buttons; after a period of $10 \mathrm{sec}$, he received the instruction that the words (or faces) list was about to begin. The subject was told to continue tapping the buttons until the experimenter instructed him to stop, which was immediately after his response to the last item on the cognitive task. Recording of the IRTs was from the time the first item appeared to the time the subject gave his final (old-new) response. In the no-concurrent memory task conditions, the subject stopped tapping at the end of $3 \mathrm{~min}$ at the experimenter's instruction $-3 \mathrm{~min}$ was the average time it would take for a memory list to be presented. In these conditions, the subject was still required to look at the memory apparatus at eye level in front of him and not allowed to look at his hands. The aim of this requirement was to keep these conditions similar in all other respects to the concurrent task conditions. For the no-concurrent manual task conditions (words alone, faces alone), the subject was instructed to keep his hands stationary and respond old or new, as appropriate. Responses from the duplicated conditions were combined so that eight sets of dependent variables were obtained from each subject under the eight experimental treatment conditions.

\section{RESULTS}

Five sets of dependent variables were examined. These included the speed (mean IRT) and consistency (standard deviation IRT) of manual performance, and error, $\mathrm{d}^{\prime}$, and $\beta$ performance on the cognitive 
tasks. A priori $F$ tests were used to assess the specific contrasts of interest between the concurrent task conditions and the no-concurrent task conditions. It was considered justifiable, on the basis of previous experimental evidence and theoretical argument, to use one-tailed significance levels to test the predictions that the concurrent cognitive tasks would disrupt contralateral hand performance (Hays, 1963, pp. 282-296).

\section{Speed of Performance Data}

Figure 1 represents the comparisons between the no-concurrent task (NCT) and the words and faces concurrent task conditions for the left and right hands for the two (6 or $12 \mathrm{~cm}$ apart) tapping conditions.

For the no-concurrent task words comparison, the right hand was significantly disrupted by concurrent words performance for both 6- and 12-cm tapping distances $[F(1,28)=2.98, \mathrm{p}<.05$, for the 6 -cm condition, and $F(1,28)=5.03, \mathrm{p}<.01$, for the $12-\mathrm{cm}$ condition]. For the left-hand words comparisons, the left hand was not significantly affected by concurrent words performance at either the 6- or 12-cm tapping conditions $[F(1,28)=0.01$, n.s., for the $6-\mathrm{cm}$ condition, and $F(1,28)=0.39$, n.s., for the $12-\mathrm{cm}$ condition].

For the NCT-faces comparisons, the left hand was significantly disrupted by concurrent faces performance for both the $6-$ and $12-\mathrm{cm}$ tapping conditions $[F(1,28)=3.33, \mathrm{p}<.05$, for the $6-\mathrm{cm}$ condition, and $F(1,28)=3.46, p<.05$, for the $12-\mathrm{cm}$ condition]. Right-hand faces performance was not significantly different from NCT performance for either the 6- or the 12-cm tapping conditions $[F(1,28)=$ 0.55 , n.s., for the $6-\mathrm{cm}$ condition, and $F(1,28)=$

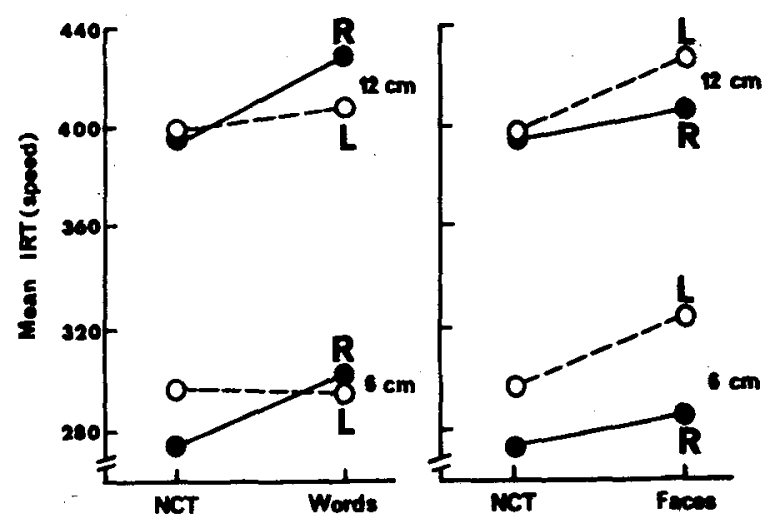

Figure 1. Changes in manual performance from the noconcurrent task (NCT) condition to the concurrent words (figure on left) and concurrent faces (figure on right) memory task conditions for the speed of performance index (mean IRT in milleseconds); these changes are presented for both the small $(6-\mathrm{cm})$ and large (12-cm) tapping distances. Closed lines present changes in right-hand performance (R); open lines present changes in lefthand performance (L).
0.47 , n.s., for the $12-\mathrm{cm}$ condition]. There was a significant decrease in overall speed of manual performance with the increased distance between the two button switches $[F(1,14)=6.53, p<.025]$.

\section{Consistency of Performance Data}

Figure 2 presents the comparisons between the NCT performance and the words and faces concurrent task conditions for the left and right hands at the two (6- or 12-cm) tapping distances. For the NCT words comparisons, the right hand was significantly disrupted by concurrent words performance for both 6- and 12-cm tapping distances $[\mathrm{F}(1,28)=15.67$, $\mathrm{p}<.001$, for the $6-\mathrm{cm}$ condition, and $F(1,28)=$ $15.26, \mathrm{p}<.001$, for the $12 \mathrm{-cm}$ condition]. For the left-hand words comparisons, the results differ from those found with the speed of performance data. There was no significant effect on left-hand performance in the $6-\mathrm{cm}$ condition $[\mathrm{F}(1,28)=1.57$, n.s. $]$. However, under the $12-\mathrm{cm}$ condition, left-hand performance was significantly disrupted by concurrent words performance $[F(1,28)=9.73, p<.01]$. Thus, at the increased tapping distance, both left- and right-hand consistency of performance was disrupted. It was considered justifiable to examine the relative changes in left- and right-hand performance for this $12-\mathrm{cm}$ condition. This interaction of Hands by NCT-Words failed to achieve significance $[\mathrm{F}(1,28)=0.31$, n.s. $]$.

For the NCT-faces comparisons, the left hand was significantly disrupted by concurrent faces performance for both the 6- and 12-cm tapping conditions $[\mathrm{F}(1,28)=8.30, \mathrm{p}<.005$, for the $6-\mathrm{cm}$ condition, and $F(1,28)=28.53, \mathrm{p}<.001$, for the $12-\mathrm{cm}$ condition]. For the $6-\mathrm{cm}$ condition, the right hand was not significantly disrupted with concurrent faces performance $[F(1,28)=2.90$, n.s.]. However, with the $12-\mathrm{cm}$ condition, the right-hand performance was significantly disrupted $[\mathrm{F}(1,28)=12.11, \mathrm{p}<.005]$. Again, it was considered justifiable to examine the relative effects and to assess the Hand by NCT-Faces interaction. However, as with the $12-\mathrm{cm}$ words performance, the interaction was not significant $[F(1,28)$ $=2.96$, n.s.]. As with the speed of performance data, there was an overall significant difference in performance between the 6 - and 12-cm tapping conditions $[F(1,14)=96.635, p<.001]$.

\section{Performance on the Words Task Conditions}

Figure 3 presents the changes in words recognition for each of the performance indices, total errors, $\mathrm{d}^{\prime}$, and $\beta$. Significant differences of concurrent words performance (word with hand) from the wordsalone condition are indicated on that figure by means of asterisks $\left({ }^{*}\right)$. Table 1 presents the obtained $F$ and $p$ values for all the comparisons. 


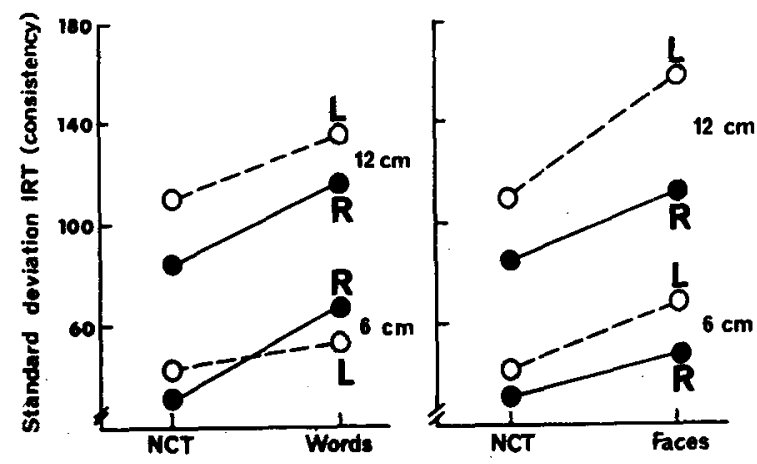

Figure 2. Changes in manual performance from the noconcurrent task (NCT) condition to the concurrent words (figure on left) and concurrent faces (figure on right) memory task conditions for the consistency of performance index (standard deviation IRT in milleseconds); these changes are presented for both the small $(6-\mathrm{cm})$ and large $(12-\mathrm{cm})$ tapping distances. Closed lines present changes in right-hand performance (R); open lines present changes in left-hand performance $(L)$.

There were no significant effects obtained under the 6-cm tapping conditions except for one change in $\beta$ which occurred for the comparison between the words alone and words with left-hand activity. Under the 12-cm tapping condition, the words error and $\mathrm{d}^{\prime}$ performance was significantly disrupted by concurrent right-hand activity. No significant change in $\beta$ was observed under this condition. No significant effects were found with concurrent left-hand activity under the $12-\mathrm{cm}$ tapping conditions.

\section{Performance on the Faces Tasks Conditions}

Figure 4 presents the changes in faces recognition performance for each of the indices, errors, $d^{\prime}$, and $\beta$. Table 2 presents the obtained $F$ and $p$ values for all the comparisons.

Significant differences occurred between the facesalone condition and the faces-plus-left-hand condition on all measures except $\beta$ under both 6 - and $12-\mathrm{cm}$ conditions. No significant changes in faces recognition performance were observed with the addition of concurrent right-hand activity (see Table 2).

\section{Comparisons Between Words and Faces Performance}

There were significant differences between the wordsalone and faces-alone conditions. Significantly more errors were made on the faces recognition task than on the words task $[F(1,28)=10.36, p<.01]$. This was due to a difference in sensitivity as assessed by $\mathrm{d}^{\prime}$ $[\mathrm{F}(1,28)=8.48, \mathrm{p}<.01]$. There was no significant difference for $\beta$, which was approximately equal to unity (optimal criterion) for both the words and faces conditions.

\section{DISCUSSION}

Increasing the tapping distance produced the most noticeable effects on the consistency of manual performance. Both the concurrent words and faces tasks produced a bilateral disruption of the hands under the $12-\mathrm{cm}$ tapping condition, but a selective disruption of the contralateral hand under the 6-cm tapping condition. These findings support the hypothesis that the selective disruption of the contralateral hand by a lateralized cognitive task is dependent upon the load imposed on those neural structures mediating the concurrent tasks. However, this hypothesis is not supported by the results obtained with the speed of performance index.

The findings with the speed of performance index indicate that, irrespective of the tapping distance requirements of the manual task, the right hand and not the left is disrupted by the concurrent words tasks and the left hand and not the right is disrupted by the concurrent faces task. These findings are consistent with those found previously (Hicks, 1975; Hicks et al., 1975; Lomas \& Kimura, 1976; McFarland \& Ashton, 1975, Note 1). Apart from a general slowing of performance, the increased tapping requirement did not
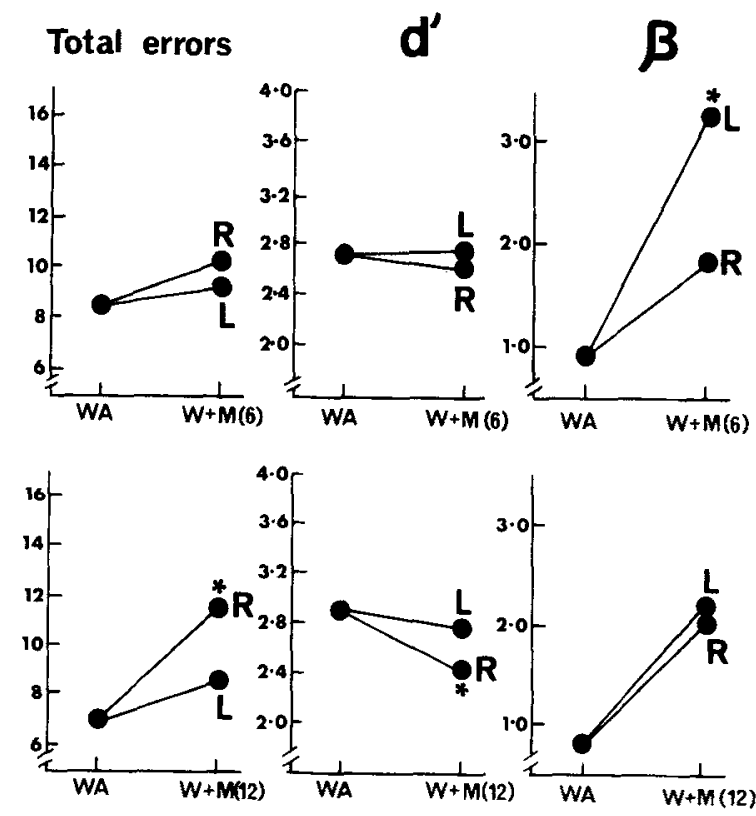

Figure 3. Changes in words recognition performance from the words alone (W A) conditions to the concurrent right- $(R)$ and left- (L) hand manual task conditions for each of the indices, total errors, $d$ ', and $\beta$. The top row of figures presents the changes in memory recognition performance with the addition of the small (6-cm) tapping task; the lower figures present the changes with the addition of the large $(12-\mathrm{cm})$ tapping task. $W+M(6$ or 12) = words plus manual task (6- or 12 -cm tapping) recognition performance. Asterisk (*) indicates that performance under this condition changed significantly when compared to words alone (W A) performance (see Table 1 ). 
Table 1

Significance Levels for Changes in Word Recognition Performance with the Addition of Concurrent Manual Activity

\begin{tabular}{cccc}
\hline Comparison & Errors & $\mathrm{d}^{\prime}$ & $\beta$ \\
\hline W A vs. W R ( 6) & $.88 \dagger$ & $.02 \dagger$ & $1.15 \dagger$ \\
W A vs. W L ( 6) & $.19 \dagger$ & $.32 \dagger$ & $9.21^{*}$ \\
W A vs. W R (12) & $7.94^{*}$ & $5.86^{* *}$ & $2.71 \dagger$ \\
W A vs. W L (12) & $.88 \dagger$ & $.57 \dagger$ & $3.31 \dagger$ \\
\hline
\end{tabular}

Note-W $A=$ words alone; $W R(6$ or 12$)=$ words with righthand, $6-$ or $12 \mathrm{~cm}$ topping; $W L(6$ or 12$)=$ words with left-hand, 6. or 12-cm tapping.

${ }^{*} p<.01 \quad{ }^{*} p<.05 \quad+$ Nonsignificant difference.

affect these patterns of selective disruption of the hands by the concurrent tasks.

The reason for obtaining different results with the speed and consistency measures of performance is not entirely clear. Perhaps the difference arose because the consistency index is more sensitive to intermittent interference effects than the speed index; the latter being a more general measure of overall performance (Michon, 1967). In this case, the different results obtained with these two indices could indicate that interference arises not only from some general excess in the overall load
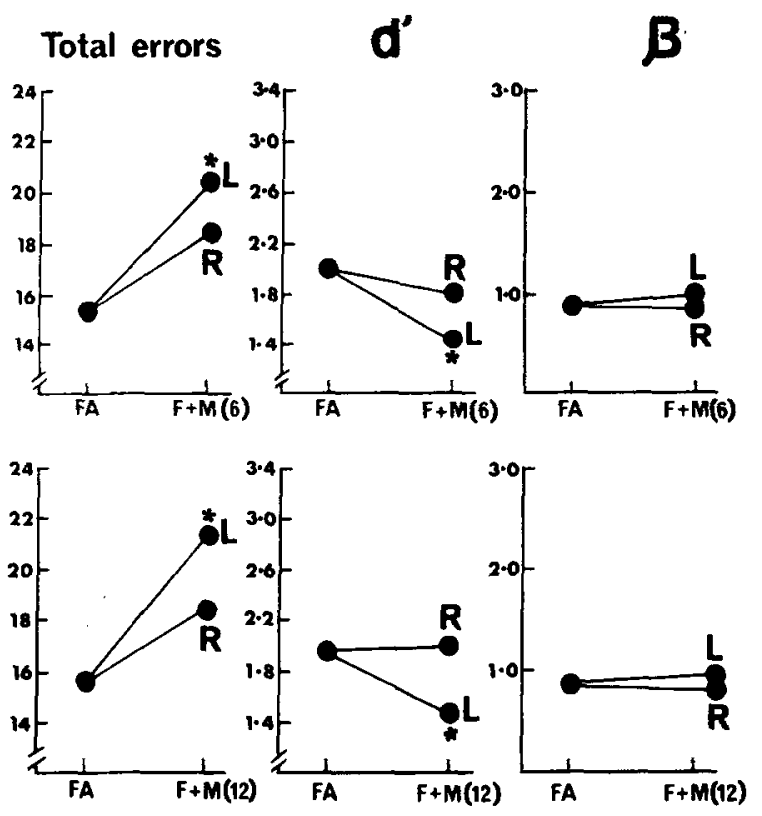

Figure 4. Changes in faces recognition performance from the faces alone (F A) conditions to the concurrent right- (R) and left- (L) hand manual task conditions for each of the indices, total errors, $d^{\prime}$, and $\beta$. The top row of figures presents the changes in memory recognition performance with the addition of the small (6-cm) tapping task; the lower figures present the changes with the addition of the large $(12-\mathrm{cm})$ tapping task. $F+M$ (6 or 12) = faces plus manual task (6- or 12-cm tapping) recognition performance. Asterisk $\left(^{\star}\right)$ indicates that performance under this condition changed significantly when compared to faces alone performance (see Table 2).
Table 2

Significance Levels for Changes in Faces Recognition Performance with the Addition of Concurrent Manual Activity

\begin{tabular}{cccc}
\hline Comparison & Errors & d' & $\beta$ \\
\hline F A vs. F R ( 6) & $2.76 \dagger$ & $1.08 \dagger$ & $.002 \dagger$ \\
F A vs. F L ( 6) & $10.57^{*}$ & $7.54^{* *}$ & $.009 \dagger$ \\
F A vs. F R (12) & $3.01 \dagger$ & $.06 \dagger$ & $.001 \dagger$ \\
F A vs. F L (12) & $7.54^{* *}$ & $4.23^{* *}$ & $.001 \dagger$ \\
\hline
\end{tabular}

Note-F $A=$ faces alone; $F R(6$ or 12$)=$ faces with right-hand, 6- or 12-cm tapping; F L (6 or 12) = faces with left-hand, 6 - or $12 . \mathrm{cm}$ tapping.

${ }^{*} p<.01 \quad * *<.05 \quad+$ Nonsignificant difference.

placed on the underlying neural structures, but also from the intermittent fluctuations in task demands across time. The implication is that the bilateral control exerted by the hemispheres over proximal activity could be intermittent in nature once some general amount of neural space had been allocated to the performance of the concurrent tasks.

The two most important findings in terms of performance on the words and faces recognition tasks were that only contralateral manual activity increased error performance, and that such changes in error performance reflected a loss of sensitivity $\left(d^{\prime}\right)$ and not a change in response bias ( $\beta$ ). Such findings are consistent with the hypothesis that interference between the concurrent tasks arises because of an overlap of the neural systems mediating task performance. The loss of sensitivity (discriminability of old and new items) would reflect a degradation of memory traces associated with the previously seen items (Lockhart \& Murdock, 1970).

The further implication of the above hypothesis is that mutual interference should occur between those tasks which are mediated by the overlapping neural systems. This was found in the present study where the disruption of the right-hand speed of performance occurred only with the concurrent words tasks, and the words performance was disrupted only by the faces task, and the faces memory performance was disrupted only by the concurrent left-hand activity. Changes in the consistency index, however, do not parallel those of cognitive performance. In line with the earlier suggestion, this may reflect qualitative differences among the dependent variables, with the speed, error, and $\mathrm{d}^{\prime}$ indices being more global measures of performance and the consistency index being a measure of intermittent effects.

The findings of the present study have a number of implications for future research. The different results obtained with the speed and consistency measures would suggest that, by only utilizing global measures of performance, important information about changes across time is lost. Future research may be profitably addressed to assessing the importance of the temporal dimension when measuring brain lateralization of func- 
tion. A second important finding of the present study was the result that mutual interference occurred only between concurrent performance of contralateral tasks. While mutual interference effects may be accounted for by a number of different types of model, this result would be a crucial prerequisite for the development of neuropsychological models framed in terms of overlapping neural systems. However, it must be stressed that the present results support only the viability of such models, not their validity.

\section{REFERENCE NOTE}

1. McFarland, K., \& Ashton, R. The influence of concurrent task difficulty on manual performance. Manuscript submitted for publication, 1977.

\section{REFERENCES}

Boll, T. J. Right and left cerebral hemisphere damage and tactile perception: Performance of the ipsilateral and contralateral sides of the body. Neuropsychologia, 1974, 12, 235-238.

CARMon, A. Impaired utilization of kinesthetic feedback in right hemisphere lesions. Neurology, 1970, 20, 1033-1038.

Carmon, A., \& Benton, A. L. Tactile perception of direction and number in patients with unilateral cerebral disease. Neurology, 1969, 19, 525-532.

Dimond, S. J., \& Beaumont, J. G. (Eds.) Hemisphere function in the human brain. London: Elek Science, 1974.

FITTs, P. M. The information capacity of the human motor system in controlling the amplitude of movement. Journal of Experimental Psychology, 1954, 47, 381-391.

Fontenot, D. J., \& Benton, A. L. Tactile perception of direction in relation to hemispheric locus of lesion. Neuropsychologia, 1971, 9, 83-88.

Hays, W. L. Statistics. New York: Holt, Rinehart \& Winston, 1963.

Hrcks, R. E. Intrahemispheric response competition between vocal and unimanual performance in normal adult human males. Journal of Comparative and Physiological Psychology, 1975, 89. 50-60.

Hicks, R. E., Provenzano, F. J., \& Rubenstein, E. D. Generalized and lateralized effects of concurrent verbal rehearsal upon performance of sequential movements of the fingers by the left and right hands. Acta Psychologia, 1975, 39, 119-130.

Kimura, D. Cerebral dominance for speech. In D. B. Towers (Ed.), The nervous system (Vol. 3). New York: Raven Press, 1975.

Kimura, D., \& ARchibald, Y. Motor functions of the left hemisphere. Brain, 1974, 97, 337-350.

KuĆERA, H., \& Francis, W. N. Computational analysis of present day American English. Rhode Island: Brown University Press, 1967.

Lockhart, R. S., \& Murdock, B. B. Memory and the theory of signal detection. Psychological Bulletin, 1970, 74, 100-109.

Lomas, J., \& KImURA, D. Intrahemispheric interaction between speaking and sequential manual activity. Neuropsychologia. 1976, 14. 23-33.

MCFARLAND, K., \& Ashton, R. The lateralized effects of concurrent cognitive activity on a unimanual skill. Cortex, 1975, 11, 283-290.

MCNicol, D. A primer of signal detection theory. London: Allen and Unwin, 1972.

Michon, J. A. Timing in temporal tracking. Soesterberg, The Netherlands: Institute for Perception RVO-TNO, 1967.

Moscovitch, M., Scullion, D., \& Christie, D. Early versus late stages of processing and their relation to functional hemisphere asymmetries in face recognition. Journal of Experimental Psychology: Human Perception and Performance, 1976, 2, 401-416.

Oldfield, R. C. The assessment and analysis of handedness: The Edinburgh Inventory. Neuropsychologia, 1971, 9, 97-113.

Shepard, R. N., \& Teghtsoonian, M. Retention of information under conditions approaching a steady state. Journal of Experimental Psychology, 1961, 62, 302-309.

WYKE, M. The effects of brain lesions on the performance of bilateral arm movements. Neuropsychologia, 1971, 9, 33-42.

(Received for publication August 16, 1977; revision accepted January 5, 1978.) 